\title{
Compact Bragg Gratings for Long-Range Surface Plasmon Polaritons
}

\author{
Boltasseva, Alexandra; Bozhevolnyi, Sergey I; Nikolajsen, Thomas; Leosson, Kristjan
}

Published in:

Journal of Lightwave Technology

Link to article, DOI:

$10.1109 / \mathrm{JLT} .2005 .862470$

Publication date:

2006

Document Version

Publisher's PDF, also known as Version of record

Link back to DTU Orbit

Citation (APA):

Boltasseva, A., Bozhevolnyi, S. I., Nikolajsen, T., \& Leosson, K. (2006). Compact Bragg Gratings for LongRange Surface Plasmon Polaritons. Journal of Lightwave Technology, 24(2), 912-918.

https://doi.org/10.1109/JLT.2005.862470

\section{General rights}

Copyright and moral rights for the publications made accessible in the public portal are retained by the authors and/or other copyright owners and it is a condition of accessing publications that users recognise and abide by the legal requirements associated with these rights.

- Users may download and print one copy of any publication from the public portal for the purpose of private study or research.

- You may not further distribute the material or use it for any profit-making activity or commercial gain

- You may freely distribute the URL identifying the publication in the public portal 


\title{
Compact Bragg Gratings for Long-Range Surface Plasmon Polaritons
}

\author{
Alexandra Boltasseva, Sergey I. Bozhevolnyi, Thomas Nikolajsen, and Kristjan Leosson
}

\begin{abstract}
By introducing periodic thickness modulation of thin metal stripes embedded in a dielectric, compact and efficient Bragg gratings for long-range surface plasmon polaritons (LR-SPPs) operating around $1550 \mathrm{~nm}$ are realized. Reflection and transmission spectra of the gratings having different lengths (from 20 to $160 \mu \mathrm{m}$ ), heights (tens of nanometers), and widths of the metal ridges forming the grating were measured, and the reflectivity of up to $60 \%$ and bandwidths ranging from 5 to $40 \mathrm{~nm}$ are demonstrated. By using a simple lossless-uniform-grating description, the effective refractive-index modulation in LR-SPP gratings is estimated to be of the order of $10^{-2}$, and two different approaches for these calculations are compared. The LR-SPP loss incurred in the investigated gratings is also discussed.
\end{abstract}

Index Terms-Integrated optics devices, multiple scattering, surface plasmons.

\section{INTRODUCTION}

$\mathbf{I}$ N RECENT years, surface-bound electromagnetic excitations propagating along the metal-dielectric interface, the so-called surface plasmon polaritons (SPPs) [1], have attracted much interest in the context of highly integrated photonic devices and nanooptics [2]. For these waves, the electromagnetic field amplitudes are strongly enhanced at the interface and decay exponentially into both of the neighboring media [1]. These features make SPPs very sensitive to surface features allowing one to efficiently control the SPP propagation in the surface plane by using different types of surface metal nanostructures [2]-[7]. However, SPPs suffer from rather strong damping due to absorption in metals (ohmic loss) so that their propagation length is limited to micrometer distances $(\sim 30 \mu \mathrm{m}$ in visible and $\sim 300 \mu \mathrm{m}$ in near infrared, for a silver-air interface). Moreover, SPPs are rather difficult to excite by the conventional end-fire coupling of radiation with single-mode fibers; and more elaborate schemes (grating or prism coupling) are to be used.

The SPP characteristics change drastically in the symmetric configuration of two very close metal-dielectric interfaces, e.g., the surfaces of a thin metal film embedded in dielectric. Such a structure supports propagation of the field-symmetric modal

Manuscript received June 21, 2005; revised September 21, 2005.

A. Boltasseva is with the Department of Communications, Optics, and Materials, COM DTU, Nano DTU, Technical University of Denmark, Kongens Lyngby DK-2800, Denmark (e-mail: aeb@com.dtu.dk).

S. I. Bozhevolnyi is with the Department of Physics and Nanotechnology, Aalborg University, Aalborg Øst DK-9220, Denmark (e-mail: sergey@ physics.aau.dk).

T. Nikolajsen is with the Crystal Fibre A/S, Birkerød DK-3460, Denmark (e-mail: tni@crystal-fibre.com)

$\mathrm{K}$. Leosson is with the Science Institute, University of Iceland, Reykjavik IS-107, Iceland (e-mail: k.leosson@ raunvis.hi.is).

Digital Object Identifier 10.1109/JLT.2005.862470 solution, the so-called a long-range SPP (LR-SPP), whereby a coupling of SPPs at two metal-dielectric interfaces results in a very small field concentration within the central metal film and, hence, low propagation loss for the LR-SPP [8]-[10]. Furthermore, for a metal stripe embedded in the dielectric, the dimensions of the metal stripe can be chosen so that the LR-SPP field distribution matches that of a single-mode fiber, facilitating the efficient end-fire excitation with the coupling loss being less than $1 \mathrm{~dB}$ per facet [11], [12].

LR-SPP stripe waveguides thereby constitute a new alternative for integrated optical (IO) circuits with one unique feature-the possibility of using the same metal-stripe circuitry for both guiding optical radiation and transmitting electrical signals that control its guidance. Different LR-SPP-based passive IO components, including straight and bent waveguides, Y-splitters, and directional couplers, have been recently experimentally demonstrated [12], [13]. In addition, efficient LR-SPP-based dynamic devices with low power consumption, including various modulators and switches, have been also realized utilizing the thermooptic effect in the polymer cladding [14], [15]. After different passive and active LR-SPPbased components for IO have been demonstrated, it seems natural to study Bragg gratings based on LR-SPP-supporting configurations, having in mind great interest in Bragg gratings for broad range of applications in distributed feedback (DFB) and distributed Bragg reflection (DBR) lasers, Bragg filters, (de)multiplexers, etc. Our preliminary investigations have verified the feasibility of realization of efficient Bragg gratings for LR-SPP stripe waveguides [16]. Recently, another approach for making Bragg gratings for LR-SPPs, which is different from the one described in [16] and here, was reported [17].

In this paper, we report in detail on the fabrication and optical characterization at telecom wavelengths of compact LR-SPP ridge gratings formed by the periodical thickness modulation of metal stripes and present a simple analysis of the results obtained. We investigate the LR-SPP transmission through and reflection from gratings with different parameters at telecom wavelengths and compare the performance of the gratings with the behavior of a lossless uniform grating. The paper is organized as follows. In Section II, the fabrication procedure for making metal ridge gratings is described. Section III is devoted to the experimental part of our work. First, the experimental setup is described. Secondly, experimental spectra for the LR-SPP transmission through and reflection from gratings are shown for different grating parameters. Experimental results for losses introduced by such gratings are also presented and discussed. Comparison with a lossless uniform grating is presented in Section IV, where the effective refractive-index 


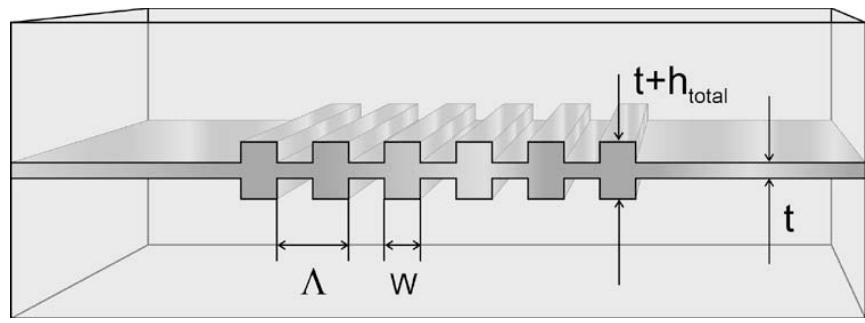

Fig. 1. Schematic (side view) of the ridge grating for LR-SPPs formed by periodic symmetric ridges protruding from a metal film embedded in dielectric.

modulation in investigated metallic gratings is calculated using two different methods. Finally, we summarize the results and offer our conclusions in Section V.

\section{DESIGn AND FABRiCATION}

The investigated grating structures for LR-SPPs were fabricated using the electron-beam lithography (EBL). First, a silicon substrate was first spin coated with a $15-\mu \mathrm{m}$-thick benzocyclobutene (BCB) polymer layer followed by soft baking of the polymer. Prior to the EBL, the wafer with polymer cladding was coated with positive electron beam followed by resist prebaking. Then the designed grating pattern was transferred into the resist by EBL exposure and development processes.

In order to transfer the pattern into the $\mathrm{BCB}$ layer, a reactive ion etching (RIE) technique was used. After RIE of the $\mathrm{BCB}$ layer with the electron-beam resist as a mask, ridges were formed by metal (gold) deposition [deposited gold thickness $h_{\text {total }}$ (see Fig. 1) from 10 to $60 \mathrm{~nm}$ ] and lift-off. Since vertical symmetry of the structure is very important for the LR-SPP propagation [8] and scattering, the etch depth was measured using an atomic force microscope (AFM). This allowed to choose the appropriate gold thickness as doubled etch depth to obtain ridges of different heights $\left(h_{\text {total }} / 2\right)$ placed symmetrically above and below the LR-SPP propagation plane. After metal deposition, the profile of the gold ridges was studied using AFM [Fig. 2(a)] in order to obtain the precise values of the ridge heights on each side of the waveguide plane [28 nm above and $32 \mathrm{~nm}$ below for the structure in Fig. 2(a) where $60 \mathrm{~nm}$ of gold was deposited] as well as to estimate the roughness of the metal surface (of the order of a few namnometers). For fabricated grating structures, the asymmetry of the ridges with respect to the waveguide plane was below $15 \%$. 40- $\mu \mathrm{m}$-long ridges of different widths from 125 to $375 \mathrm{~nm}$ (filling factors, defined as a ratio between the ridge width and the grating period, $w / \Lambda$, from 0.25 to 0.75 ) were arranged in 500-nm-period gratings of different lengths having from 20 to 160 periods. For the approximate value $n \approx 1.53$ of the $\mathrm{BCB}$ refractive index found in the $\mathrm{BCB}$ data sheet [18], this period $(\Lambda)$ was expected to result in the efficient reflection occurring at the Bragg wavelength $\lambda_{B} \approx 2 n \Lambda \approx 1.53 \mu \mathrm{m}$. After the grating fabrication, LR-SPP stripe waveguides, placed in the middle of the gratings [Fig. 2(b)], were patterned using standard ultraviolet (UV) lithography. Gratings were made wide enough (the length of the ridges, as mentioned above, was $40 \mu \mathrm{m}$ ) to ensure the full overlap with the LR-SPP mode propagating along the 8 - $\mu \mathrm{m}$-wide stripe and to increase tolerance for aligning the
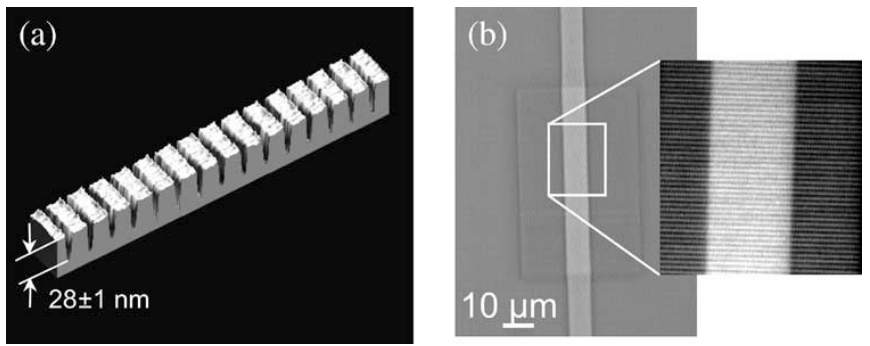

Fig. 2. (a) Profile of the ridges on one side of the waveguide plane obtained using atomic force microscopy (the total height of the ridges is approximately 60 to $32 \mathrm{~nm}$ below the waveguide plane and $28 \mathrm{~nm}$ above) together with the (b) optical microscope image showing the top view of the $40-\mu \mathrm{m}$-wide and $80-\mu \mathrm{m}$-long ridge grating placed in the middle of an $8-\mu \mathrm{m}$-wide stripe.

UV mask with stripe waveguides with respect to EBL-patterned gratings. A second metallization and lift-off was used to pattern gold stripes, being approximately $15 \mathrm{~nm}$ in thickness $(t)$ (see Fig. 1) and typically $8 \mu \mathrm{m}$ in width, which ensured low-loss LR-SPP propagation and efficient radiation (in/out) coupling with single-mode fibers [11], [12]. The last processing step consisted of spin coating the wafer with another $15-\mu \mathrm{m}$-thick BCB layer. After final polymer curing, the wafer was cut into individual samples.

\section{EXPERIMENTAL RESUlts}

\section{A. Experimental Arrangement}

Optical characterization of the fabricated LR-SPP gratings was carried out using standard spectrally resolved transmission and reflection measurements (Fig. 3). The light from a broadband source based on edge-emitting light-emitting diode (EE-LED) $(1.55 \mu \mathrm{m})$ was sent through a polarization controller (to adjust the electric field polarization perpendicular to the stripe-waveguide plane) and launched into the LR-SPP waveguide via butt coupling from a polarization-maintaining single-mode fiber. In order to strip off all light coupled into the fiber cladding, $1 \mathrm{~km}$ of coiled standard single-mode fiber was used as out-coupling fiber. The output signal was detected by an optical spectrum analyzer.

The grating transmission spectra were normalized to the reference stripe transmission, thereby canceling out the propagation and coupling losses. The reflection spectra were normalized to the reflection from the mirror and corrected by the approximate values of coupling and propagation loss for LR-SPP waveguides. This was done in the following manner. First, the coupling loss was measured independently for $8-\mu \mathrm{m}$ wide stripe waveguides (which did not contain any gratings on them) and found to be $0.6 \pm 0.1 \mathrm{~dB}$ per facet. After that, for each set of the grating parameters (ridge height, filling factor, and grating length), the reflection spectra from four identical gratings placed at four different distances $(2,4,6$, and $8 \mathrm{~mm})$ from the input plane to the grating were measured. This allowed to estimate the value of the propagation loss for each particular sample that was found to be of the order of $8 \mathrm{~dB} / \mathrm{cm}$. As a final step, for each set of the grating parameters, the reflection from a grating placed $2 \mathrm{~mm}$ from the input plane was first corrected by the reflection from the mirror (100\% reflection), 


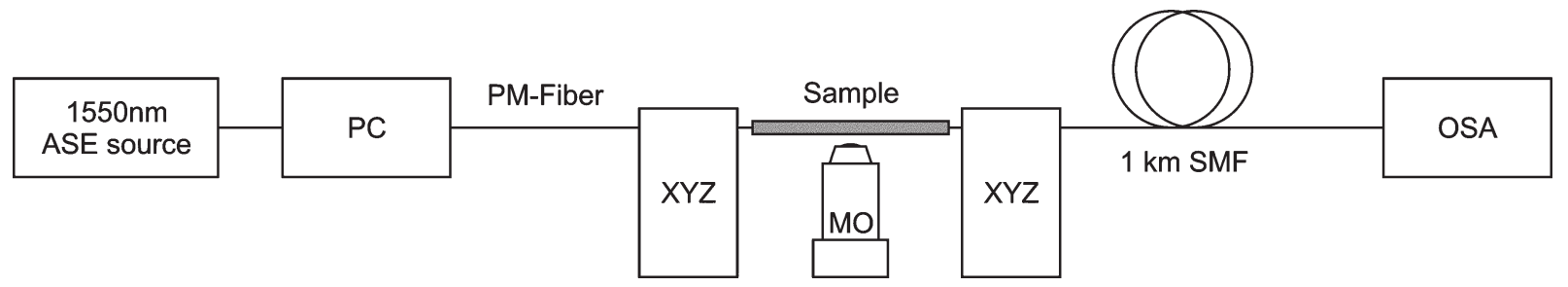

Fig. 3. Scheme of the experimental arrangement for the reflection and transmission measurements (PC: polarization controller; OSA: optical spectrum analyzer).

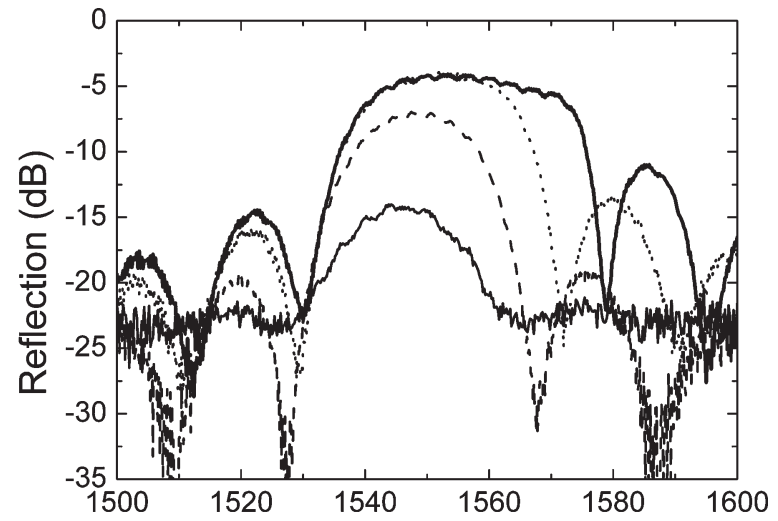

(a)

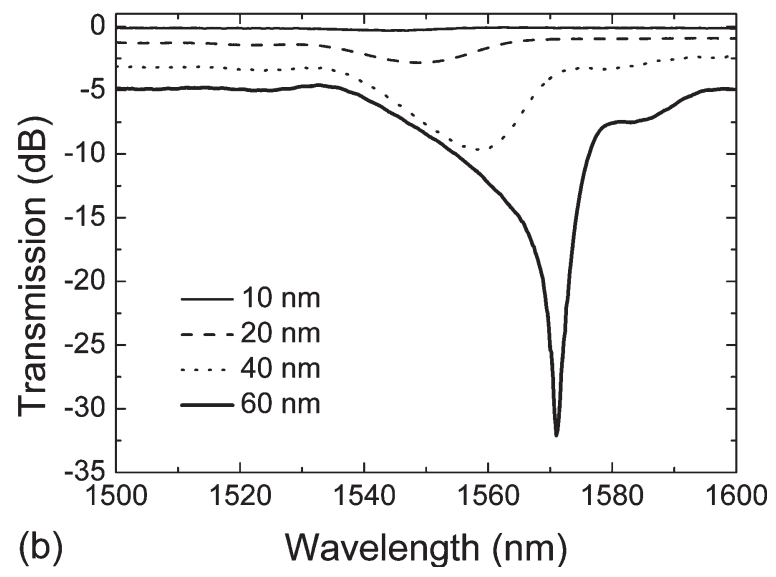

Fig. 4. Measured (a) reflection and (b) transmission spectra for the 40- $\mu$ mlong gratings with different heights of the ridges $\left(h_{\text {total }}\right)$. Grating period is $500 \mathrm{~nm}$ and filling factor is 0.45 .

then the correction was made for doubled coupling loss and the propagation loss over $4 \mathrm{~mm}$ of the stripe waveguide.

\section{B. Reflection and Transmission Spectra}

Typical reflection and transmission spectra are presented in Figs. 4 and 5 for different heights of the ridges and two different grating lengths. All in all, the investigated gratings showed the behavior expected from conventional dielectric Bragg gratings [19], with the Bragg wavelength being close to that estimated above (a small offset is most probably related to the difference in the BCB refractive index from the data sheet value as discussed in [16]). It is also seen that, for the total ridge height below $40 \mathrm{~nm}$, the grating strength is proportional to the ridge height (Figs. 4 and 5). For such low ridges, the gap in transmission and the peak in reflection increase with the increase of the ridge height. As seen in Figs. 4 and 5, one can increase

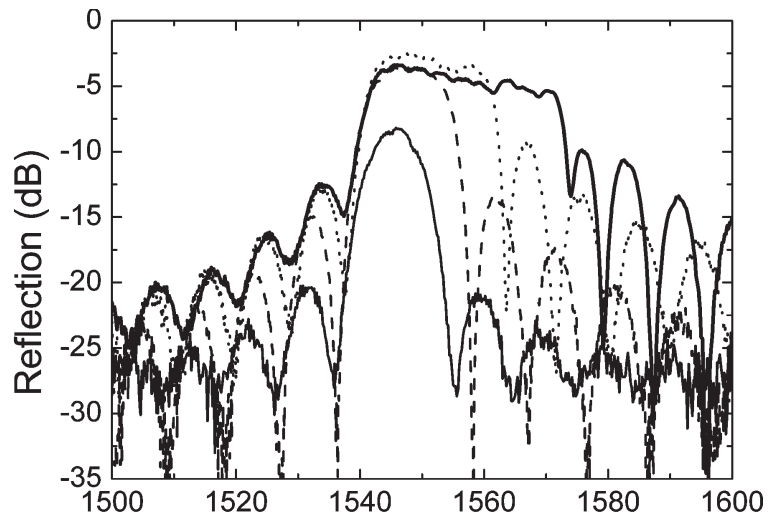

(a)

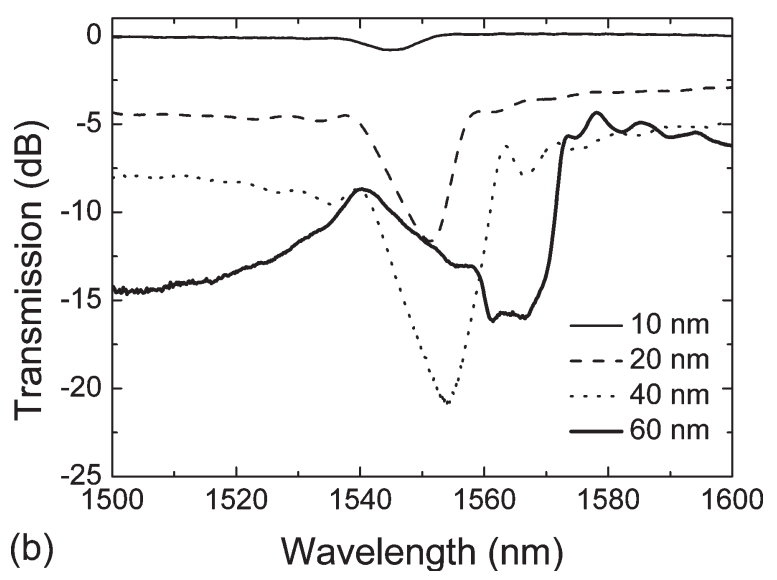

Fig. 5. Measured (a) reflection and (b) transmission spectra for the $80-\mu \mathrm{m}-$ long gratings. All else is as in Fig. 4.

the grating strength either by increasing the grating length or making higher ridges (increasing the effective refractiveindex modulation). With the increase of grating length, the maximum reflection gradually increases until it reaches saturation (Fig. 6). At the same time, the transmission dip also evolves with the grating length, exhibiting progressively larger contrast. However, due to simultaneously increasing absorption and scattering losses, the transmission level decreases at all wavelengths - also outside the Bragg resonance. For the same grating length, the gratings become stronger when increasing the ridge height that leads to both larger effective refractiveindex modulation and larger insertion (scattering and absorption) losses, resulting, in turn, in broadening of the reflection peak and faster saturation (Fig. 6). Thus, for the total ridge height of more than $40 \mathrm{~nm}$, the maximum reflection never exceeds $60 \%$ due to the fact of increased absorption and scattering loss for high ridges, where the out-of-plane scattering 


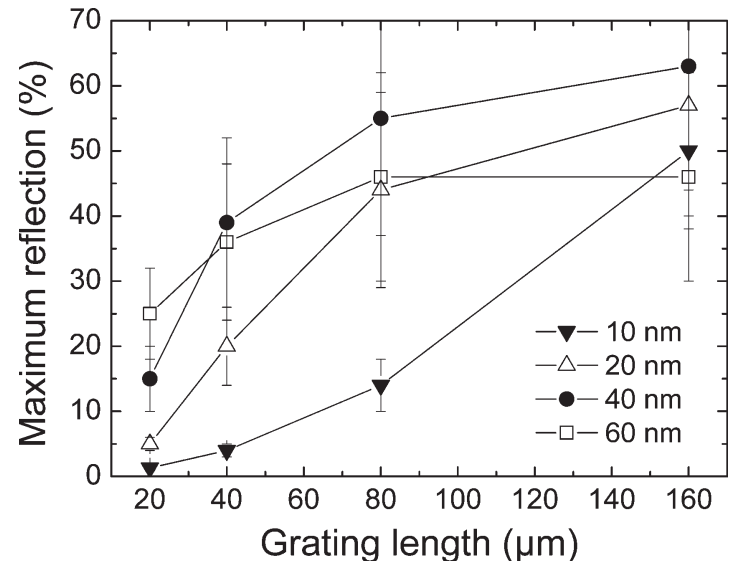

Fig. 6. Maximum reflection versus grating length for different heights of the ridges $\left(h_{\text {total }}\right)$. All else is as in Fig. 4.

gradually takes over and both transmission and reflection characteristics become progressively distorted (Figs. 4 and 5).

One of the characteristic features in the behavior of the investigated gratings is an asymmetry in the spectra with respect to the Bragg wavelength. The asymmetry is getting more pronounced with the increase of heights of the ridges (Figs. 4 and 5). Due to the asymmetry, the Bragg wavelength was found to shift into shorter wavelengths with the increase in the grating length [16]. This asymmetry is thought to be related to two different loss mechanisms in such gratings-absorption in metal and out-of-plane scattering. These two effects were described in more detail and compared with the results of theoretical modeling in [16], where it was shown that the loss introduced by the grating is dominated by the out-of-plane scattering. The latter can be decomposed into the coherent scattering, i.e., the directional LR-SPP diffraction out of the plane by the grating, and the diffuse LR-SPP scattering by individual metal ridges. The first process removes the radiation out of the propagation plane only for the wavelengths below the Bragg wavelength, while the diffuse scattering occurs at all wavelengths (though it is expected to gradually increase toward shorter wavelengths) [16]. The described difference in loss contributions below and above the Bragg wavelength gives rise to different transmission loss levels outside the Bragg condition observed for all investigated gratings (Figs. 4 and 5).

The increase in the grating length not only increases its maximum reflectivity at the Bragg wavelength but also narrows the spectral width of Bragg resonance, at least for relatively low ridges (cf. Figs. 4 and 5). The interplay between the grating length and the resonance bandwidth for different ridge heights is most transparent in the dependence of the bandwidth-length product that is expected to be constant, i.e., $\delta \lambda L \sim\left(\lambda_{B} / n\right)^{2}$ $\sim 1 \mu \mathrm{m}^{2}$, if the efficient Bragg diffraction occurs over the whole grating length [16]. The bandwidth-length product is, however, expected to increase once the grating length becomes longer than the LR-SPP penetration (inside the grating) length at the Bragg wavelength. It is clear that, for gratings with higher ridges, this increase should begin for shorter grating lengths, as indeed was observed in our experiments (Fig. 7). Finally, we would like to emphasize that changing the total ridge height from 10 to $60 \mathrm{~nm}$ and gratings length from 20 to

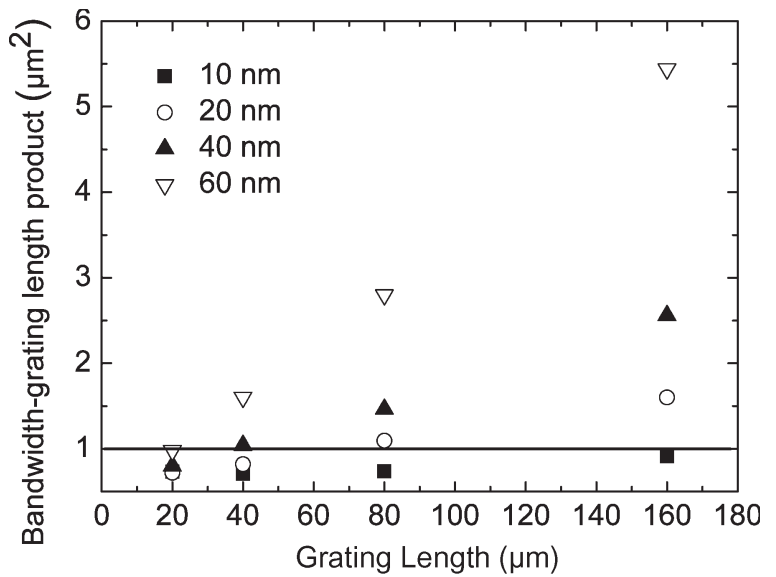

Fig. 7. Dependence of the product between the reflection peak width and the grating length on the length of the grating for different heights $\left(h_{\text {total }}\right)$ of the ridges. Grating period is $500 \mathrm{~nm}$ and filling factor is 0.55 .

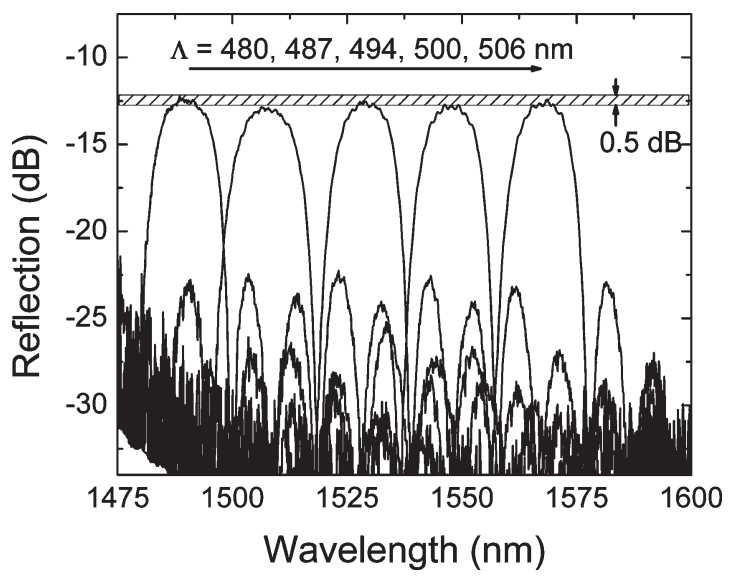

Fig. 8. Reflection from the $80-\mu \mathrm{m}$-long gratings of different periods. The total ridge height $\left(h_{\text {total }}\right)$ is $20 \mathrm{~nm}$ and the filling factor is 0.35 .

$160 \mu \mathrm{m}$ allowed realization of the very broad range of grating performance-from weak gratings with a bandwidth of only $5 \mathrm{~nm}$ for low ridges up to very strong and broadband gratings with approximately $40 \mathrm{~nm}$ of bandwidth.

The reflection spectra were also recorded for five gratings of the same length and ridge height but with different periods changing from 480 to $506 \mathrm{~nm}$. These spectra showed very reproducible shifts in the Bragg wavelength according to the Bragg condition (Fig. 8). The level of the maximum reflection stayed within $0.5 \mathrm{~dB}$ for all five different-period gratings (Fig. 8), demonstrating very good reproducibility.

\section{Loss Introduced by Grating}

In order to study losses introduced by metal ridge gratings, the spectral dependences of the reflection $(R)$ and transmission $(T)$ were plotted together with the total power $(T+R)$ spectrum (Fig. 9). The most pronounced and somewhat unexpected feature, which was observed for all investigated gratings is that the loss level at the Bragg wavelength was found to be smaller than the loss levels on both sides of the Bragg condition. The observed decrease in the total loss for the Bragg wavelength can be due to both reduced out-of-plane scattering and reduced 


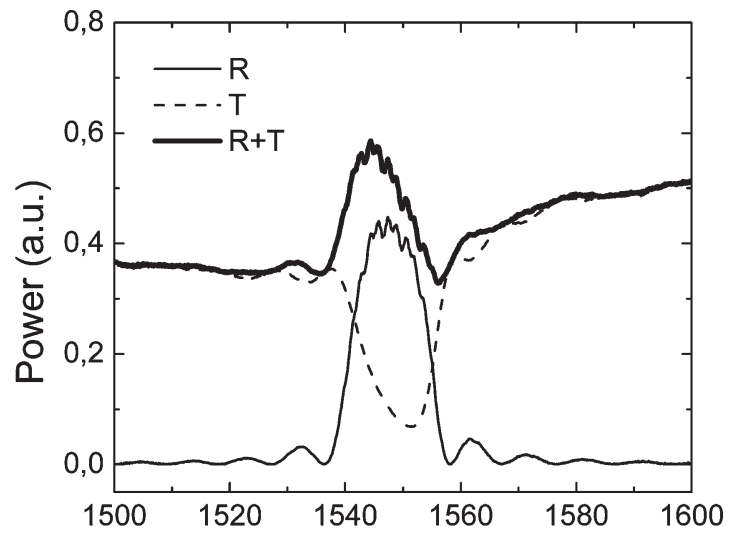

(a)

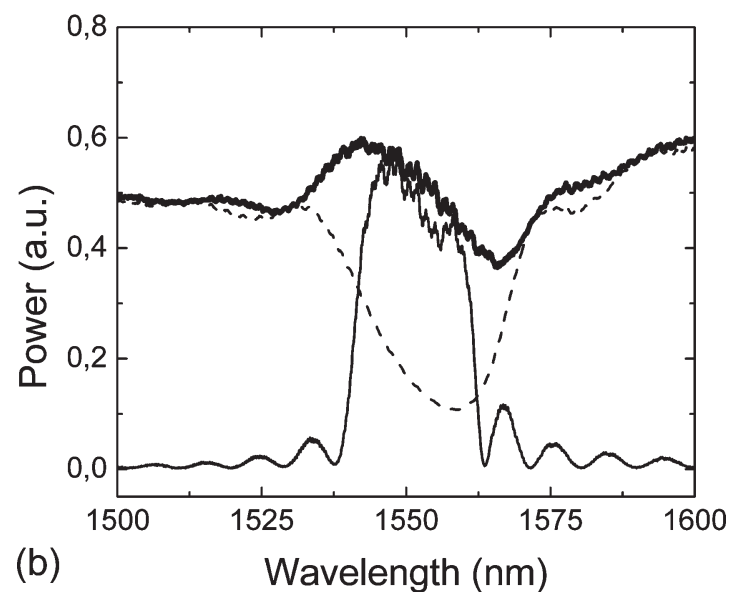

Fig. 9. Reflection $(R)$, transmission $(T)$, and total power $(R+T)$ spectra for (a) 80- $\mu \mathrm{m}$-long grating with a total ridge height $\left(h_{\text {total }}\right)$ of $20 \mathrm{~nm}$ and (b) $40-\mu \mathrm{m}$-long grating with a total ridge height $\left(h_{\text {total }}\right)$ of $40 \mathrm{~nm}$. Grating period is $500 \mathrm{~nm}$ and filling factor is 0.45 .

absorption in metal. The decrease in the out-of-plane scattering might be connected to the situation when the standing field pattern at the resonance has minima at the ridge edges giving less scattering. As for the decrease in the absorption loss, this can be related to the fact that at the resonance, the field does not penetrate that deep into the grating as at the wavelengths outside the resonance; and less overlap between the LR-SPP field and metal ridges gives less absorption. Moreover, the roughness and imperfection of ridges may also contribute similarly to the absorption loss.

The loss introduced by a grating was found to increase with the length of the grating and the height of the ridges both at the Bragg wavelength and outside the resonance due to both increasing out-of-plane scattering and absorption in metal (Fig. 10). For example, at $1500 \mathrm{~nm}$ for a $40-\mu \mathrm{m}$-long grating, the grating loss increased from $\sim 0.5 \mathrm{~dB}$ for a total ridge height of $10 \mathrm{~nm}$ to $\sim 14 \mathrm{~dB}$ for 60 -nm-high ridges.

There is a tradeoff between the grating strength and loss introduced by the grating; and for a particular grating performance, it is always possible to choose optimal parametersfor example, ridge height and filling factors that give the most compact grating. It is seen, for example, from the dependencies shown in Fig. 6 that by making ridges higher $(40 \mathrm{~nm}$ instead of $20 \mathrm{~nm}$ ), one can obtain the same grating strength for a shorter grating (40 $\mu \mathrm{m}$ long instead of $80 \mu \mathrm{m})$.
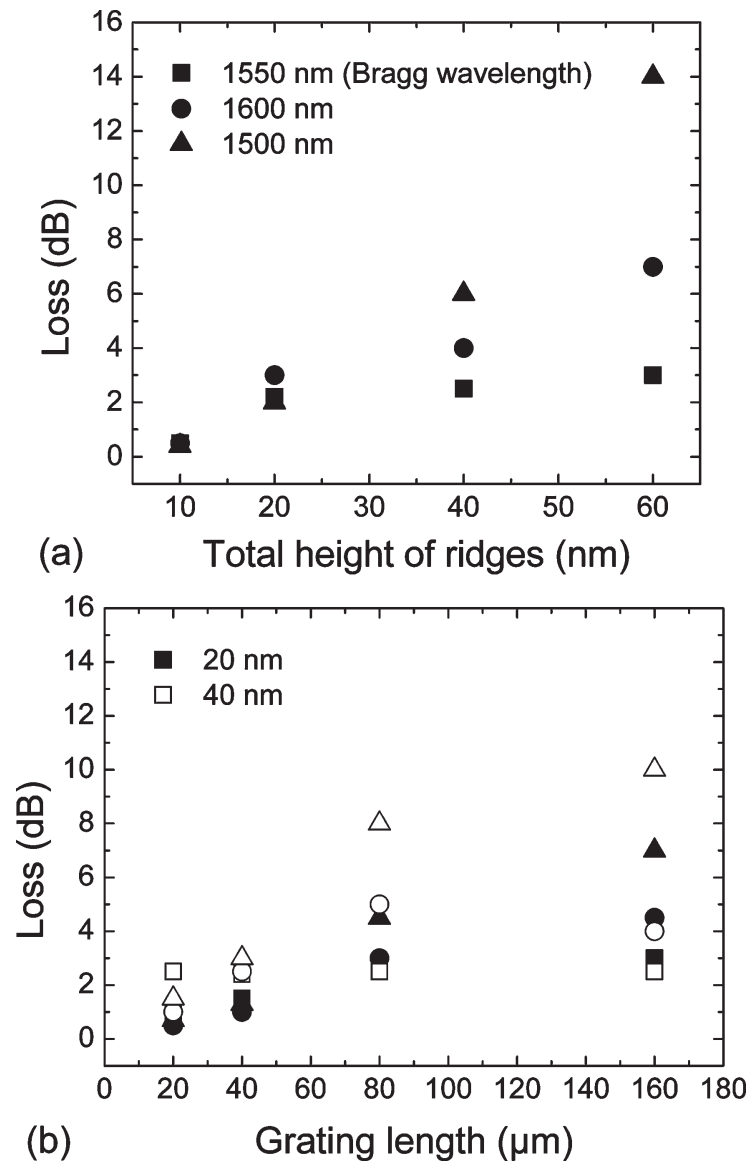

Fig. 10. Loss introduced at the Bragg wavelength and wavelengths below and above the resonance for (a) different heights $\left(h_{\text {total }}\right)$ of the ridges $(40-\mu$ m-long gratings) and (b) different grating lengths ( $h_{\text {total }}$ of 20 and $40 \mathrm{~nm}$ ). Grating period is $500 \mathrm{~nm}$ and filling factor is 0.35 .

\section{COMParison With LOSSLESS Uniform GRating}

In order to estimate the effective refractive-index modulation in LR-SPP gratings, a simple approach based on comparison with a lossless uniform Bragg grating is used. In this simple model, the effective refractive-index modulation $\Delta n$ can be estimated from the maximum reflection $R_{\max }$ using the expression [20]

$$
R_{\text {max }}=\tanh ^{2}\left(\frac{2 \Delta n L}{\lambda_{B}}\right)
$$

where $\lambda_{B}$ is the Bragg wavelength and $L$ is the length of the grating. Using this expression, the effective refractive-index modulation can be easily found from the experimental reflection spectra. However, since the maximum reflection, which can be achieved for a given ridge height, is limited by the loss introduced by the grating, this approach can only be relied upon for low ridge heights. For high ridges, when the loss contribution is significant, this method will give understated values of $\Delta n$.

Another way of estimating the effective refractive-index modulation is through reflection spectra zero points. The peak reflectivity that defines the maximum reflectivity at the Bragg wavelength can also be written as [21]

$$
R_{\max }=\tanh ^{2}(\kappa L)
$$




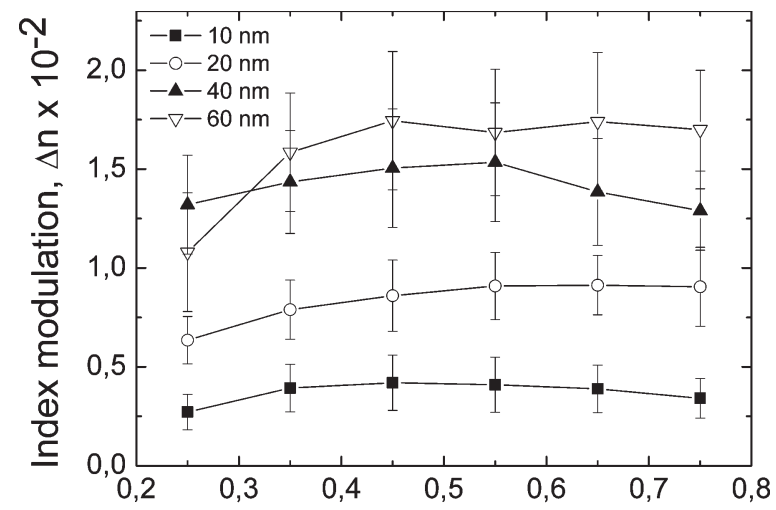

(a)

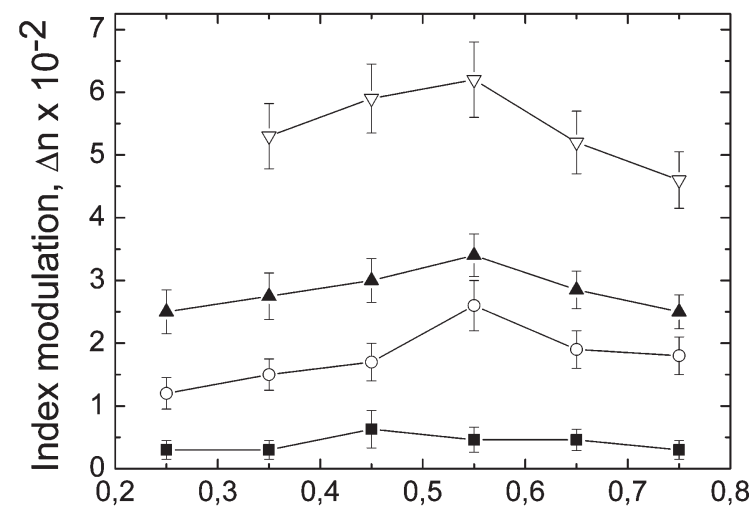

(b)

Fill factor

For low ridges (total height of $10 \mathrm{~nm}$ ), both methods give the same value for the effective refractive-index modulation of about $0.3 \times 10^{-2}$. For higher ridges, $\Delta n$ estimated from the maximum reflection is lower than the one calculated using the reflection spectra zeros, a circumstance that is, as already mentioned, connected to the fact of grating loss increasing with the ridge height.

The experimental data revealed that the grating characteristics and, thereby, the effective refractive-index modulation depended rather weakly on the filling factor. Thus, the estimated $\Delta n$ was only about $20 \%-30 \%$ higher for the filling factors around 0.5 compared to the $\Delta n$ for the lowest/highest filling factors (Fig. 11).

It is interesting to notice that when using a one-dimensional mode solver by Kymata Software (previously used in [12] to study LR-SPP propagation characteristics for infinitely wide stripes), the difference between effective refractive indices for the LR-SPP modes propagating along metal films of thicknesses $t$ and $t+h_{\text {total }}$ (see Fig. 1) was found to be of the same order of magnitude as the effective refractive-index modulation estimated above (for example, for the total ridge height of $10 \mathrm{~nm}$, the difference between effective refractive indices (LR-SPPs on 15- and 25-nm-thick gold films) is $0.26 \times 10^{-2}$ compared to $\sim 0.3 \times 10^{-2}$ estimated using maximum reflection/reflection zeros).

\section{Discussion And Conclusion}

Fig. 11. Effective refractive-index modulation calculated from (a) the maximum reflection and (b) zero points of the reflection spectra versus filling factor for different heights $\left(h_{\text {total }}\right)$ of the ridges.

where $\kappa$ is often called the reflectivity per unit length or the grating strength and is directly related to the effective refractive-index modulation $\Delta n$ (1). For strong gratings $(\kappa L>1)$, the spectral dependence of the reflection has a plateau-like shape where the reflectivity of the grating is very high. In this case, the position $\lambda_{m}$ of the $m$ th zero point of the reflection spectrum is given by

$$
\lambda_{m}=\lambda_{B}\left(1+m \frac{\Lambda}{L} \sqrt{\left(\frac{|\kappa| L}{m \pi}\right)^{2}}+1\right), \quad m= \pm 1,2 \ldots
$$

The grating strength (and, therefore, the effective refractiveindex modulation) can thereby be estimated from the two firstorder zero points $\lambda_{+1}(m=+1)$ and $\lambda_{-1}(m=-1)$ as

$$
|\kappa| L=\pi \sqrt{\left(\frac{\lambda_{+1}-\lambda_{-1}}{\lambda_{+1}+\lambda_{-1}} \cdot \frac{L}{\Lambda}\right)^{2}-1}
$$

This method is more reliable in case of lossy gratings than the previous one, since it does not use the absolute value of the maximum reflection and, therefore, is not that influenced by the loss.

The comparison between two methods of determining the effective refractive-index modulation is presented in Fig. 11.

In summary, we have studied the LR-SPP propagation along periodically thickness-modulated metal stripes embedded in dielectric for light wavelengths in the telecom range, demonstrating that the efficient Bragg gratings can be achieved by making shallow (tens of nanometers) metal ridges. The investigated LR-SPP gratings were found to behave, to some extent, similar to conventional reflecting Bragg gratings [19], giving the possibility of very simple design and parameter choice. Even for low ridges (total ridge height of 10-20 nm), the LRSPP penetration depth was estimated to be $\sim 100 \mu \mathrm{m}$ at the resonance wavelength (Fig. 7), corroborating the possibility of realizing strong and compact gratings based on such thicknessmodulated metal stripes. It should be noted that in another approach for making Bragg gratings for LR-SPPs, viz., by varying the width of a metal stripe supporting the LR-SPP propagation [17], modulation of the LR-SPP propagation is considerably weaker than that achieved in our work (by varying the stripe thickness), so that the gratings in [17] had to be considerably longer (a few millimeters), exhibiting very narrow bandwidths (below $1 \mathrm{~nm}$ ). In order to achieve very large modulation depths (0.1) and full reflection within about $10 \mu \mathrm{m}$ of grating length in this alternative configuration proposed in [17], one can use a thicker metal along with the metal-gap design. However, in this case, increased modulation depth comes not only with wider bandwidths, but also with increased propagation loss along the stripe LR-SPP waveguide. We believe that these two approaches are somewhat complementary with different application potentials.

The use of LR-SPP Bragg gratings gives an advantage of choosing many grating parameters (for example, grating size 
and bandwidth) in a very broad range, which, for example, cannot be done in case of fiber Bragg gratings due to the photorefractive effect limitation. Moreover, the fabrication procedure for making LR-SPP gratings is quite robust and has very good reproducibility (see Fig. 8). As for the intrinsic "onepolarization" property of the LR-SPP grating, we believe that for some applications (e.g., sensors based on light interference or DFB lasers), it is advantageous that the LR-SPP-based components transmit only one polarization.

In conclusion, as an alternative to the integrated Bragg gratings, LR-SPP-based gratings can provide both efficiency and compactness and can be easily integrated with couplers, splitters, and other LRSPP-based waveguide components to be used for wavelength selective functions. As we expect that many advances in the LR-SPP-related research are to be made in the coming years, a great potential in the devices based on Bragg gratings involving LR-SPPs is foreseen for a variety of applications ranging from lasers with DFB and wavelength filters to biosensors.

\section{REFERENCES}

[1] H. Raether, Surface Plasmons. Berlin, Germany: Springer-Verlag, 1988.

[2] W. L. Barnes, A. Dereux, and T. W. Ebbesen, "Surface plasmon subwavelength optics," Nature, vol. 424, no. 6950, pp. 824-830, Aug. 2003.

[3] I. I. Smolyaninov, D. L. Mazzoni, and C. C. Davis, "Imaging of surface plasmon scattering by lithographically created individual surface defects," Phys. Rev. Lett., vol. 77, no. 18, pp. 3877-3880, Oct. 1996.

[4] S. I. Bozhevolnyi and F. A. Pudonin, "Two-dimensional micro-optics of surface plasmons," Phys. Rev. Lett., vol. 78, no. 14, pp. 2823-2826, Apr. 1997.

[5] H. Ditlbacher, J. R. Krenn, G. Schider, A. Leitner, and F. R. Aussenegg, "Two-dimensional optics with surface plasmon polaritons," Appl. Phys. Lett., vol. 81, no. 10, pp. 1762-1764, Sep. 2002.

[6] S. I. Bozhevolnyi, J. Erland, K. Leosson, P. M. W. Skovgaard, and J. M. Hvam, "Waveguiding in surface plasmon polariton band gap structures," Phys. Rev. Lett., vol. 86, no. 14, pp. 3008-3011, Apr. 2001.

[7] S. I. Bozhevolnyi, V. S. Volkov, and K. Leosson, "Localization and waveguiding of surface plasmon polaritons in random nanostructures," Phys. Rev. Lett., vol. 89, no. 18, pp. 186801-186804, Oct. 2002.

[8] D. Sarid, "Long-range surface-plasma waves on very thin metal films," Phys. Rev. Lett., vol. 47, no. 26, pp. 1927-1930, Dec. 1981.

[9] J. J. Burke, G. I. Stegeman, and T. Tamir, "Surface-polariton-like waves guided by thin, lossy metal films," Phys. Rev. B, Condens. Matter, vol. 33, no. 8, pp. 5186-5201, Apr. 1986.

[10] P. Berini, "Plasmon-polariton waves guided by thin lossy metal films of finite width: Bound modes of symmetric structures," Phys. Rev. B, Condens. Matter, vol. 61, no. 15, pp. 10484-10503, Apr. 2000.

[11] T. Nikolajsen, K. Leosson, I. Salakhutdinov, and S. I. Bozhevolnyi, "Polymer-based surface-plasmon-polariton stripe waveguides at telecommunication wavelengths," Appl. Phys. Lett., vol. 82, no. 5, pp. 668-670, Feb. 2003.

[12] A. Boltasseva, T. Nikolajsen, K. Leosson, K. Kjaer, M. S. Larsen, and S. I. Bozhevolnyi, "Integrated optical components utilizing longrange surface plasmon polaritons," J. Lightw. Technol., vol. 23, no. 1, pp. 413-422, Jan. 2005.

[13] R. Charbonneau, N. Lahoud, G. Mattiussi, and P. Berini, "Demonstration of integrated optics elements based on long-ranging surface plasmon polaritons," Opt. Express, vol. 13, no. 3, pp. 977-984, Feb. 2005.

[14] T. Nikolajsen, K. Leosson, and S. I. Bozhevolnyi, "In-line extinction modulator based on long-range surface plasmon polaritons," Opt. Commun., vol. 244, no. 1-6, pp. 455-459, Jan. 2005.

[15] — - "Surface plasmon polariton based modulators and switches operating at telecom wavelengths," Appl. Phys. Lett., vol. 85, no. 24, pp. 5833-5835, Dec. 2004.

[16] S. I. Bozhevolnyi, A. Boltasseva, T. Søndergaard, T. Nikolajsen, and K. Leosson, "Photonic band gap structures for long-range surface plasmon polaritons," Opt. Commun., vol. 250, no. 4-6, pp. 328-333, Jun. 2005.

[17] S. Jetté-Charbonneau, R. Charbonneau, N. Lahoud, G. Mattiussi, and P. Berini, "Demonstration of Bragg gratings based on long-ranging surface plasmon polariton waveguides," Opt. Express, vol. 13, no. 12, pp. 4674-4682, Jun. 2005.

[18] CYCLOTENE Advanced Electronics Resins: Refractive Index vs. Wavelength (Dow Chemical Company). [Online]. Available: http://www.dow. com/cyclotene/solution/refwave.htm

[19] A. Yariv and P. Yeh, Optical Waves in Crystals. New York: Wiley, 1984.

[20] L. A. Coldren and C. W. Corzine, Diode Lasers and Photonic Integrated Circuits. New York: Wiley-Interscience, 1995.

[21] T. E. Murphy, "Design, fabrication and measurement of integrated Bragg grating optical filters," Ph.D. thesis, Dept. Elect. Eng. Comput. Sci., Massachusetts Inst. Technol., Cambridge, 2001.

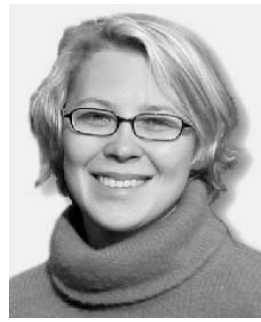

Alexandra Boltasseva received the M.Sc. degree in physics from the Moscow Institute of Physics and Technology, Moscow, Russia, in 2000, and the Ph.D. degree in electrical engineering from the Technical University of Denmark (DTU), Lyngby, Denmark, in 2004.

She was previously with Micro Managed Photons A/S, Farum, Denmark, and Alight Technologies A/S. She is now a post-doctoral researcher at the Department of Communications, Optics, Materials, DTU. Her research focuses on surface-plasmon-polariton optics and advanced nanotechnology.

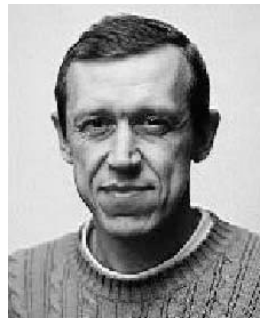

Sergey I. Bozhevolnyi received the M.Sc. degree in physics and the Ph.D. degree in quantum electronics from the Moscow Institute of Physics and Technology, Moscow, Russia, in 1978 and 1981, respectively, and the Dr.Sc. degree from Aarhus University, Aarhus, Denmark, in 1998.

From 1981 to 1990, he was an Associate Professor with the Yaroslavl Technical University, Yaroslavl, Russia. After working at the Institute of Microelectronics, Russian Academy of Sciences, as a Head of the sector "Optical technologies" from 1990-1991, he started his research on near-field optics at the Institute of Physics, Aalborg University, Aalborg, Denmark, where he has been a Professor since 2003. Previously, he was the CTO of Micro Managed Photons, Farum, Denmark. His research interests include linear and nonlinear nano-optics, surface plasmon polaritons and non-plasmonic circuits, multiple light scattering, including photonic band gap and light localization phenomena, and integrated and fiber optics.

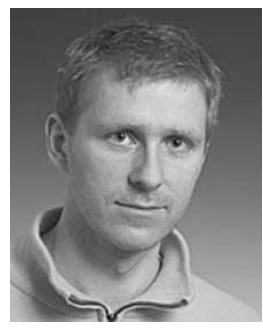

Thomas Nikolajsen received the Ph.D degree in optical engineering from the Technical University of Denmark, Lyngby.

He began his career at NP Photonics, where he specialized in Erbium-doped fiber amplifiers and then moved back to his home country, where he spent three years at Micro Managed Photon A/S, Farum, Denmark, working with plasmon-based integrated optics. Currently, he is responsible for the development of photonic crystal-based high-power laser assemblies at Crystal Fiber A/S.

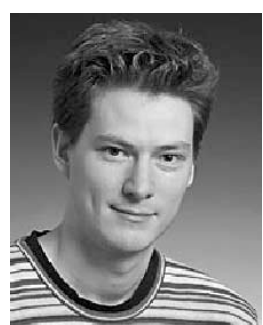

Kristjan Leosson received the B.Sc. degree in engineering physics from Queen's University, Kingston, ON, Canada, in 1994, the M.Sc. degree in technical physics from the University of Iceland, Reykjavik, Iceland, in 1996 and the Ph.D. degree in electrical engineering from the Technical University of Denmark, Lyngby, Denmark, in 2001.

He has worked as a project manager and process development engineer at Micro Manage Photons A/S, Farum, Denmark, and Lumiscence A/S, Denmark, and is now a senior research scientist at the University of Iceland, focusing on fabrication of micro- and nanoscopic structures and devices. 